\title{
Generating $F_{0}$ contours from ToBI labels using linear regression
}

\author{
Alan W Black $\quad$ Andrew J Hunt ${ }^{2}$ \\ ATR Interpreting Telecommunications Laboratories, \\ 2-2 Hikaridai, Seika-cho, Soraku-gun, Kyoto 619-02, JAPAN. \\ awb@cstr.ed.ac.uk, hunt@winston.east.sun.com
}

\begin{abstract}
This paper describes a method for generating Fo contonrs from ToBI labelled utterances. The method uses linear regression to predict $F_{0}$ target values for the start, mid-vowel and end of every syllable, using features representing the ToBI labels, stress and syllable position. Contours generated by this method for an English database have a correlation of 0.62 and $34.8 \mathrm{Ez}$ RMS error when compared with originals from test data. These results are significant improvements on a previous rule driven method $(0.40$ and 44.7$)$, and the new method contours are preferred by human listeners. The technique has also been successfully applied to Japanese ToBI with similar improvements.
\end{abstract}

\section{INTRODUCTION}

One problem in the process of synthesizing natural sounding speech is the prediction of an Fo contour which adequately reflects the desired prosodic tune. In most synthesizers the task of generating a prosodic tune consists of two sub-tasks, the prediction of intonation labels (accents, tones, etc) from text and the generation of a contour from those labels (and possibly other information). This paper deals solely with the second of those tasks.

The experiments presented here look at one particular intonation phonological labelling system and improve on an existing method of generating an $F_{0}$ contour from these labels. The ToBI labelling system [7] offers a method for labelling pertinent aspects of intonation in speech. Although there are recognized limitations with the system, it has been used to hand-label large speech databases and is being used in a number of synthesis systems.

This work has been fully implemented in ATR's CHATR speech synthesis system [2], thus showing the new technique not only produces better $F_{0}$ contonrs from hand-labelled natural utterances but also for fully synthesized utterances.

1 Now with Centre for Speech Technology Research, University of Edinburgh, Scotland.

2 Now with Sun Microsystems Laboratories, Chelmsford, MA.

\section{RULE-DRIVEN METHOD}

The ToBI labelling system has its origins in the Pierrehumbert intonation labelling system [6]. ToBI in fact has been interpreted in a number of slightly varying ways but could be generally defined as follows. A ToBI labelling for an utterance consists of three tiers each related (through time) to a speech waveform. The tiers are: labels, breaks indices and miscellaneous. The label tier marks pitch accents, phrase accents and boundary tones. The break index tier marks one of four levels of prosodic breaks. The miscellaneous tier may contain any other labelling, such as background noise, coughing, laughing, disfluencies or anything else that might be labelled.

From the synthesis point of view, the question is how well can we predict a $F_{0}$ contour using the labeis and breaks. Although useful information may exist in the miscellaneous layer, it is not formally defined what exists (and what does not) therefore it is ignored.

One method for generating an Fo contour from such labels and breaks is described in [1], which we will call the $A P L$ method. A similar generation method for a Japanese version of ToBI is described in more detail in [5, chap7]. We will briefly describe the APL method as it is this we wish to improve on.

The APL method predicts a number of target points for each syllable marked with a pitch accent, phrase accent or boundary tone. A number of specific rules deal with each case. For example consider the following diagram

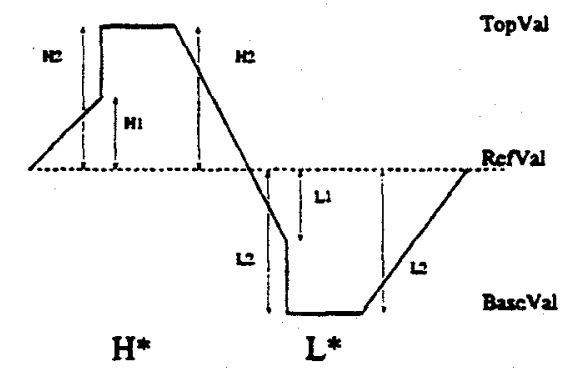

An H* accent introduces three target points, the first of 
height $\mathrm{B} 1$ above the reference line at the start of the syllable, the second at height $\mathrm{B} 2$ also at the start and the third also at $\mathrm{B2}$ at the end of the syllable. Similarly for L* except they are below the reference line.

The parameters H1, $\mathrm{H} 2$ etc. are given as fractions of TopVal and BaseVal above or below RefVal, so there is some independence from absolute pitch range. Independently RefVal TopVal and BaseVal may decrease over time to represent declination.

After all targets for labelled syllables are predicted they are smoothed to produce a more familiar $F_{0}$ looking something like

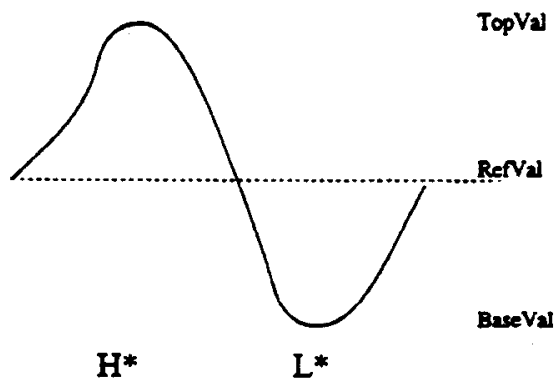

Special rules are required for syllables labelled with multiple labels, such as accents and ending tones, causing the targets to be squeezed appropriately.

The various parameters may be set by hand and experimentation (though some experiments to extract these values from data are described in [5, chap7]). For most implementations these values are set by hand and tuned until acceptable results are achieved.

\section{LINEAR REGRESSION METHOD}

Instead of trying to adjust the parameters for the model described above we are interested in antomatically finding the optimal values of these parameters. Although some form of gradient descent algorithm conld be used for optimization, a more simple quicker approach was undertaken. The approach was simply to predict three $F_{0}$ target values for every syllable, one at the start of the syllable, one in mid-vowel and one at the end of the syllable. Prediction uses the formula

$$
\text { target }=I+w_{1} f_{1}+w_{2} f_{2}+w_{2} f_{2}+\ldots+w_{n} f_{n}
$$

Where $f_{i}$ are features that are felt to contribute to the $F_{0}$ value, such as accent type, position in phrase etc. $I$ and the weights $w_{1-n}$ are estimated from data using linear regression.

Accents (i.e. ToBI pitch accents) are represented by 5 binary features, each representing the group an accent falls within. The accent groups are: accent_1: H*, accent_2: !H*, accent_3: $L *$, accent_4: $L+H * L+! H * H+! H * L *+H \quad L *+H$, accent_5: other. The complex accents are grouped becanse of their frequency within our database is low, if more data were available these could easily be split.
Phrase accents ( $\mathrm{H}-$ and $\mathrm{L}-$ ) and boundary tones are grouped together as the grammar of ToBI does not allow them to co-occur. They are grouped into 6 classes: endtone_l: $\mathrm{H}-$, end tone 2: $L-$, endtone_3: $L-L \%$, endtone_4: $L-H \%$, endtone_5: H-L\%, endtone_6: other.

The third ToBI related set of features encodes break indices. Instead of encoding the break index as a single value we encode it as 4 separate binary features, depending on which break index ( 1 to 4 ) this allows a certain amount of nonlinearity.

Thus for each syllable we collected the following features

the accent type on this syllable and that of the previous two syllables and following two syllables.

the endtone type on this syllable and the previous two syllables and following two syllables.

the break index type on this syllable and the previous two syllables and following two syllables.

the lexical stress of this syllable, and the two previous and two following syllables.

the number of syllables from start and to end of current phrase

the number of stressed syllables from start and to end of current phrase

the number of accented syllables from start and to end of current phrase

the number of syllable since last accented syllable (and to next accent)

Using the same set of features for each syllable we build three linear regression models predicting the start $F_{0}$, mid-vowel $F_{0}$ and end Fo respectively.

In generation the predicted targets are smoothed and interpolated to give a continnous contour which is applied to the waveform using PSOLA [3].

\section{COMPARATIVE RESULTS}

The above model was tested on the Boston University FM Radio corpus [4] for speaker f2b. F2b consists of about 45 minutes of female American news reading speech. It has been hand-labelled with ToBI labels, though the documentation admits there may be some inconsistencies in the labelling. The data used in this experiment consists of 14,778 syllables around $67 \%$ of which are nnaccented, $20 \% \mathrm{~B} *, 5 \% ! \mathrm{H} *, 4 \%$ $L+H *, 1.6 \% L+! H *, 1.3 \%$ and others $1 \%$.

In predicting an $F_{0}$ target value it is necessary to decide what such a value might be during unvoiced segments. As it is the contour we are trying to predict it was decided to use an interpolated contour over the whole utterance (except during panses). This was done for primarily three reasons: first as a full contour is, in onr system, presented to our prosodic modification module (PSOLA based), second smoothing a set of target points where some are forced to zero for phonetic 
reasons would be complex, and third we did not wish to include phonetic properties in our contour prediction which would require significantly more data.

For our training data we first extracted a raw contour using a standard pitch tracker (ESPS's get fo). Using the $F_{0}$ values and the vocing information we constrneted onr smoothed contour by finding the mean $F_{0}$ for voiced sections of all segments and interpolating between them (ignoring any seg. ment with no voiced sections at all). A 10 ms frame by frame comparison between the smoothed contour and raw contonr (ignoring frames marked unvoiced in the raw contour) offers an RMS error of $9.9 \mathrm{~Hz}$ with a correlation of 0.90 . Note that time alignment is not a concern. As original durations are used throughout, both contours will always be aligned.

From this training data we extracted the start, mid-vowel and end values for each syllable. We split ont data into training and test data (12000/2778) and built three linear regression models for the training data. For the individual models we achieved the following results

\begin{tabular}{|l||c|c||c|c||}
\hline \multicolumn{1}{|c||}{} & \multicolumn{2}{c||}{ Train } & \multicolumn{2}{c|}{ Test } \\
\hline & RMS & Corr & RMS & Corr \\
\hline start & 27.1 & 0.53 & 27.4 & 0.55 \\
\hline mid-v & 26.2 & 0.66 & 26.1 & 0.68 \\
\hline end & 27.7 & 0.56 & 28.4 & 0.55 \\
\hline
\end{tabular}

In order to find out if these results are an improvement we wish to compare them with the contours produced by the API method described above. We cannot directly compare them as the APL method does not explicitly predict start, mid-vowel and end target points therefore we took the IR model's results and interpolated between them and compared the continnous contours as predicted by the LR method and the APL method with the smoothed Fo we used for the LR model training. The results were

\begin{tabular}{|l||r|r||}
\hline & RMS & Corr \\
\hline APL & 44.7 & 0.40 \\
\hline IR & 34.8 & 0.62 \\
\hline
\end{tabular}

These figures suggest that the LR method is producing a contour much closer to our smoothed original.

As a further test, we implemented this algorithm within CHATR and synthesized a number of utterances from the test set using the APL and LR models. In each case we took the original "natural" information from the utterance (i.e. segments, durations, ToBI labels etc.) and used it to predict the Fo contour, Then we used PSOLA to impose this contour on the original natural atterance. 10 (short) sentences from the test set were chosen and played to three native English speakers (not including the authors). In $70 \%$ of the cases listeners preferred the IR Fo contonrs over the API generated ones.
The following examples offer a comparison between the techniques, and the original. Because our PSOLA implementation introduces distortion, we include the original similarly distorted to offer a fair comparison. Note the waveform example contains the full sentence while the graphs, for ease of reading only contain the first clause "Jeff Conley heads the Boston Finance Commission,"

The following graph shows the original smoothed Fo [SOUND A803S01.WAV]

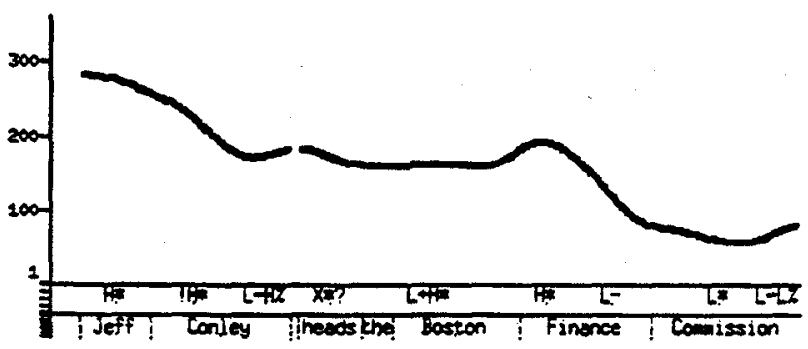

The contour generated by APL method for the same ntterance shows a much more varied contour [SOUND A803S02.WAV

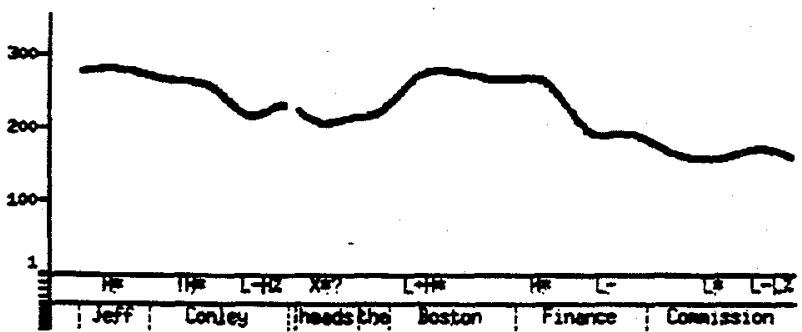

While the LR method produces [SOUND A803S03.WAV]

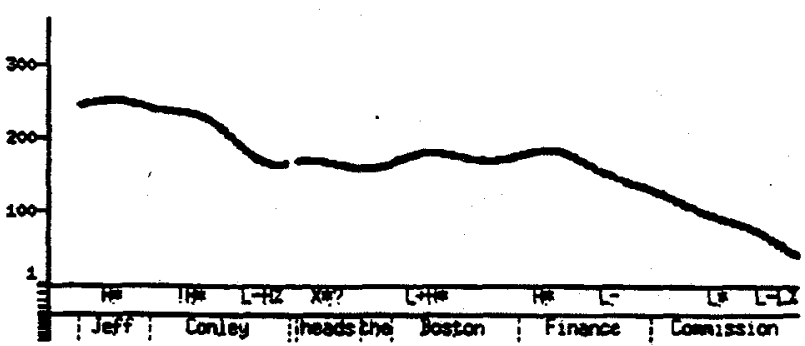

In general LR produced Fo contours were less varied than those generated by APL, sometimes cansing them to sound not as "interesting" as the APL counterpart. However the APL generated contours often sounded over-varied and inappropriate (something the LR ones never did). A more detailed critical comparison is included below

\section{JAPANESE TOBI}

To further test this LR model we applied it to a Japanese databases marked with Japanese ToBI (JToBI) as described in [5]. Using the same technique we nsed a database of 503 sentences spoken by a male (Tokyo) Japanese speaker (ATR MET Bset). We compared the result against a previously 
existing implementation of that described in [5] which atthough caters for the different intonational phonology (i.e. for Japanese) is effectively similar to the API technique for English described above. The following results were achieved for full generated contours when compared with the smoothed original.

\begin{tabular}{|l||c|c|}
\hline & RMS & Corr \\
\hline API & 25.6 & 0.55 \\
\hline LR & 20.9 & 0.70 \\
\hline
\end{tabular}

The results were played to a Japanese native speaker and although in general expressed a preference for the LR sentences, the API sentences were never particularly bad. The better results for Japanese are probably due to a number of simple reasons. First Japanese intonation is probably less varied than English, the Japanese speaker (MHT) is very consistent, MHT's pitch range is less than $\{2 b$ 's. Also more time was spent tuning the Japanese APL parameters than for English. But it should be noted that the Japanese parameters were tuned over a year while the LR model was trained and applied in an afternoon. It gives better results and is immediately customizable to other speakers.

\section{DISCUSSION}

From the above results it appears that the linear regression method better models an $F_{0}$ contour. It is fully trainable and shows improvement over previous techniques even for multiple languages. The implementation of the LR method is substantially simpler than the APL method. The APL method requires devising particular shapes for the various ToBI labels and creating parameters to define the size and position of these shapes. Also the APL method includes a separate feature for declination. All the parameters used to realise these features need to be given values, so far in our implementations, by hand, although some training method could be devised. In the IR case it is simply a matter of collecting the feature values for each feature and summing their weighted values, where the weights are explicitly available from the training method.

When a ToBI labelled database is not available for training the results from a different speaker, of the same dialect, may be transfered. Absolute $F_{0}$ target values from the trained model are converted into zscores (i.e. number of standard deviations from the $F_{0}$ mean). Those zscores may be converted into the target speaker's range using the $F_{0}$ mean and standard deviation of the target speaker. This technique (which can also be used for the API model) has proved quite adequate.

However in spite of the advantages there are distinct disadvantages of this technique too. There is no way this technique will learn contours for labels not in the training database, or labels with few examples. Particularly, in the English case, the f2b news database contains only three $\mathrm{H}-\mathrm{H} \%$ boundary tones. This is insufficient for the model to learn about final rises and hence when presented with a syllable marked with $\mathrm{H}-\mathrm{H} \%$ the resulting $\mathrm{F}_{0}$ does not rise. This problem does not exist when using the APL method where explicit rules for each label are devised. A second problem is in syllables with multiple labels, where the intonation contour cannot necessarily be captnred by three target points alone. Such phenomena are rare in news speech though are more common in say dialogue speech.

Although we can assume that in databases of dialogue speech there will be more examples of the labels such as $\mathrm{H}-\mathrm{H} \%$, and likewise databases will have labels representing their intonational variation, the above $L R$ method may in fact be too general. A more specific model may give better resuits, (especially in cases where there are only a few examples of particular labels). We can look at the API model and LR model on a scale. The API model requires specific rules for each accent (and combination) that can exist on a syllable. The IR model however treats all syllables in the same way irrespective of their labels, i.e. effectively a single rule. Some medium may be better where specific label types (or clusters of labels) have specific patterns of targets. As yet this space of possible methods has not yet been investigated.

In conclusion, the results show that the linear regression method presented here offers a better modelling of the $F_{0}$ contour than previous published method, also the model does not require special rules for each label type. The model is general enough for both English and Japanese.

\section{REFERENCES}

1. M. Anderson, J. Pierrehumbert, and M. Liberman. Synthesis by rule of English intonation patterns. In Proceedings of ICASSP 84, pages 2.8.1-2.8.4, 1984.

2. A. W. Black and P. Taylor. CHATR: a generic speech synthesis system. In Proceedings of COLING-94, volume II, pages 983-986, Kyoto, Japan, 1994.

3. Eric. Moulines and Charpentier F. Pitch-synchronous waveform processing techniques for text-to-speech synthesis using diphones. Speech Communication, 9(5/6):453467, 1990.

4. M. Ostendorf, P. Price, and S. Shattuck-Hufnagel. The Boston University Radio News Corpus. Technical Report ECS-95-001, Electrical, Computer and Systems Engineering Department, Boston University, Boston, MA, 1995.

5. J. Pierrehumbert and M. Beckman. Japanese Tone Structure. The MIT Press, Cambridge, Mass., 1988.

6. Janet B. Pierrehumbert. The Phonology and Phonetics of English Intonation. $\mathrm{PhD}$ thesis, MIT, 1980. Published by Indiana University Linguistics Club.

7. K. Silverman, M. Beckman, J. Pitrelli, M. Ostendorf, C. Wightman, P. Price, J. Pierrehumbert, and J. Hirschberg. ToBI: a standard for labelling English prosody. In Proceedings of ICSLP92, volume 2, pages 867$870,1992$. 